\title{
Mercury and psychosocial stress exposure interact to predict maternal diurnal cortisol during pregnancy
}

\author{
Hannah MC Schreier ${ }^{1 *}$, Hsiao-Hsien Hsu' ${ }^{2}$, Chitra Amarasiriwardena ${ }^{2}$, Brent A Coull ${ }^{3,4}$, Lourdes Schnaas ${ }^{5}$, \\ Martha María Téllez-Rojo ${ }^{6}$, Marcela Tamayo y Ortiz ${ }^{7}$, Rosalind J Wright ${ }^{1}$ and Robert O Wright ${ }^{2}$
}

\begin{abstract}
Background: Disrupted maternal prenatal cortisol production influences offspring development. Factors influencing the hypothalamic-pituitary-adrenal axis include social (e.g., stressful life events) and physical/chemical (e.g., toxic metals) pollutants. Mercury (Hg) is a common contaminant of fish and exposure is widespread in the US. No prior study has examined the joint associations of stress and mercury with maternal cortisol profiles in pregnancy.

Objectives: To investigate potential synergistic influences of prenatal stress and $\mathrm{Hg}$ exposures on diurnal cortisol in pregnant women.

Methods: Analyses included 732 women (aged $27.4 \pm 5.6$ years) from a Mexico City pregnancy cohort. Participants collected saliva samples on two consecutive days (mean $19.52 \pm 3.00$ weeks gestation) and reported life stressors over the past 6 months. Hg was assessed in toe nail clippings collected during pregnancy.

Results: There were no main effects of $\mathrm{Hg}$ or psychosocial stress exposure on diurnal cortisol ( $p s>$.20) but strong evidence of interaction effects on cortisol slope (interaction $\mathrm{B}=.006, \mathrm{SE}=.003, p=.034$ ) and cortisol at times 1 and 2 (interaction $\mathrm{B}=-.071, \mathrm{SE}=.028, p=.013 ; \mathrm{B}=-.078, \mathrm{SE}=.032, p=.014$ ). Women above the median for $\mathrm{Hg}$ and psychosocial stress exposure experienced a blunted morning cortisol response compared to women exposed to higher stress but lower Hg levels.
\end{abstract}

Conclusions: Social and physical environmental factors interact to alter aspects of maternal diurnal cortisol during pregnancy. Research focusing solely on either domain may miss synergistic influences with potentially important consequences to the offspring.

Keywords: Mercury, Psychosocial, Stress, Pregnancy, Cortisol, HPA axis

Cortisol is a hormone that is secreted by the adrenal cortex following activation of the hypothalamic-pituitary-adrenal (HPA) axis. Typically, cortisol secretion follows a diurnal pattern, such that cortisol levels are low overnight, begin to rise just before awakening, peak shortly after waking ( 3045 minutes), and subsequently decline throughout the day [1]. Cortisol plays a critical role in early development $[2,3]$ and disruptions in prenatal maternal cortisol production may play a role in neurodevelopmental disorders, including impaired cognitive development, autism, and behavioral

\footnotetext{
* Correspondence: hannah.schreier@mssm.edu

'Department of Pediatrics, Icahn School of Medicine at Mount Sinai, One Gustave L. Levy Place, Box 1198, New York, NY 10029, USA

Full list of author information is available at the end of the article
}

problems among offspring [4]. Consequently, understanding environmental contributors to altered maternal cortisol rhythms during pregnancy represents an important step towards preventing adverse outcomes in offspring.

Endocrine assessment, including glucocorticoid production, has been used in the detection of early or low-level effects of environmental stressors as a means of identifying individuals at risk for later health problems. The HPA axis is subject to potential disruption by both nonchemical (social stressors) and chemical factors [5-8].

Previous research has shown that both the experience of acute and chronic psychological stressors is associated with altered cortisol output $[8,9]$. In general, adverse experiences (i.e., stress) with a chronic or cumulative

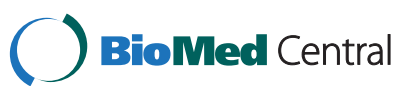

(c) 2015 Schreier et al.; licensee BioMed Central. This is an Open Access article distributed under the terms of the Creative Commons Attribution License (http://creativecommons.org/licenses/by/4.0), which permits unrestricted use, distribution, and reproduction in any medium, provided the original work is properly credited. The Creative Commons Public Domain Dedication waiver (http://creativecommons.org/publicdomain/zero/1.0/) applies to the data made available in this article, unless otherwise stated. 
nature most strongly influence child health risk, perhaps by producing a more sustained (i.e., trait-like) shift in HPA axis functioning. Moreover, social environmental factors, including greater life stress and stress correlates (e.g., anxiety, depression), have been linked to altered diurnal cortisol production among pregnant women [10-12]. Stress-elicited physiological responses vary and are influenced by characteristics of the stressor (e.g., magnitude, timing, chronicity). Under chronic stress, the HPA axis may operate at higher or lower levels compared to normal homeostasis [8]. Due to the complexity of cortisol metabolism, disruptions of homeostasis may manifest in a variety of ways, such as lower cortisol at awakening, an altered morning rise, or a slower decline over the day. Each in turn has been linked with adverse health outcomes. For example, altered prenatal cortisol production in pregnant mothers, particularly elevated levels later in the day, has been associated with adverse child outcomes including low birth weight, poorer cognition, and repeated wheeze [13-15].

In parallel, a toxicology literature on cortisol disruption has developed. Exposure to toxic metals, such as mercury $(\mathrm{Hg})$, is also being implicated in the alteration of HPA axis functioning [16] in animal studies on mammals and fish [17-20]. Hg is a heavy metal that, in organic form, e.g. methylmercury $(\mathrm{MeHg})$, is readily absorbed by the gastrointestinal tract following ingestion [21]. Hg pollution in the environment is converted to $\mathrm{MeHg}$ by bacteria and because $\mathrm{MeHg}$ is poorly excreted, it bioaccumulates up the food chain with higher concentrations found in larger predatory animals, including predatory fish such as tuna, sharks and swordfish, and in the animals that consume them, such as humans [22]. Moreover, $\mathrm{MeHg}$ exposure has been linked to a number of adverse neurodevelopmental outcomes in humans, including reduced motor skills, neuromuscular changes, and a variety of cognitive function deficits [23,24]. $\mathrm{MeHg}$ exposure during pregnancy via maternal fish consumption has also been associated with adverse neurodevelopmental outcomes in their children, although not all studies have found deficits [25-27]. To date, no studies in humans have examined the relationship between prenatal $\mathrm{Hg}$ exposure and cortisol production in pregnant women.

Given that exposure to psychosocial stressors may lead to altered physiological states (e.g., greater inflammation, altered HPA axis functioning) that are similar to states induced by toxic chemicals, it is natural to speculate that joint exposure to both may increase an individual's vulnerability to these external stressors in the physical environment. There has been an increasing call for the joint consideration of social and physical environmental influences on health [28-33]. Several animal studies have examined joint effects of prenatal stress and other metals (e.g., lead) on HPA axis functioning $[5,34,35]$ and a few studies have examined effects of lead-stress interactions on the HPA axis in postnatal samples of older children [6,36]. To our knowledge, no study in humans has considered whether combined exposure to social stress and Hg may alter diurnal HPA axis functioning in humans synergistically.

In the present study we investigate potential synergistic influences of a social pollutant, negative life events, and a physical pollutant, $\mathrm{Hg}$ exposure, on diurnal daily cortisol rhythms in a sample of pregnant women participating in a Mexico City birth cohort. We hypothesize that the experience of negative life events and $\mathrm{Hg}$ exposure interact to alter maternal prenatal diurnal cortisol rhythms.

\section{Materials and methods Participants}

Between 2007 and 2011, women who were at 12-24 weeks' gestation and healthy, were recruited to participate in this study as part of an ongoing prospective Mexico City birth cohort. All women had to be at least 18 years of age, have access to a telephone, and plan to stay in Mexico City for at least three years following recruitment. Women with diagnoses of heart or kidney disease, who used steroid or antiepilepsy drugs, or who reported daily alcohol consumption were not eligible. There were 3898 women seen in the clinics over this time span, $84 \%$ of whom were eligible. Of these we enrolled 1054. All enrolled women signed a letter of informed consent in the late $1^{\text {st }}$ trimester and were seen for a study visit in the $2^{\text {nd }}$ trimester. Among the enrolled women the attrition rate was $30.6 \%$ due to women dropping out prior to delivery or having a spontaneous abortion ( $\mathrm{n}=93$; 8.8\%) or remaining enrolled but missing $\mathrm{Hg}$ data, psychosocial stress data, or cortisol data $(n=229$; $21.7 \%$ ) and being dropped from the analyses. These women did not differ from women who remained in the analyses with respect to socioeconomic status, body mass index, smoking during pregnancy, and parity (all $p \mathrm{~s}>.10$ ); they were marginally younger $[\mathrm{t}=-1.695$, $\mathrm{p}=.090]$. The final sample included 732 women (aged $27.4 \pm 5.6$ years). See Table 1 for a summary of participant characteristics. All participants provided written informed consent. This study was approved by the ethics boards of the Harvard School of Public Health, the Mexican National Institute of Public Health, the Mexican National Institute of Perinatology, and the Icahn School of Medicine at Mount Sinai.

\section{Mercury}

Maternal $\mathrm{Hg}$ exposure was measured in toenail clippings obtained either during the $2^{\text {nd }}(\mathrm{n}=474,64.8 \%)$ or $3^{\text {rd }}$ $(\mathrm{n}=258,35.2 \%)$ trimester of pregnancy. Total toenail $\mathrm{Hg}$ represents a longer term index of $\mathrm{Hg}$ exposure over 
Table 1 Sample descriptives

\begin{tabular}{lll}
\hline & $\mathbf{n}(\%)$ & M $( \pm$ SD) \\
\hline Age (years) & & $27.4( \pm 5.6)$ \\
BMI & & $27.0( \pm 4.3)$ \\
Highest level of education & & \\
Middle school & & \\
or less & $188(25.7 \%)$ & \\
Technical post-middle school & $107(14.6 \%)$ & \\
High school or junior college & $215(29.4 \%)$ & \\
Technical post-junior college & $32(4.4 \%)$ & \\
College & $175(23.9 \%)$ & \\
Graduate & $11(1.5 \%)$ & \\
Psychosocial stress (CRISYS) & & $0.19( \pm 0.15)$ \\
Toenail mercury ( $\mu$ g/g) & & $19.52( \pm 3.00)$ \\
Gestational age at saliva collection & & $-0.04( \pm 0.02)$ \\
Cortisol AUC (nmol/L; log) & & \\
Cortisol slope (nmol/L; log) & &
\end{tabular}

$\mathrm{BMI}=$ body mass index; CRISYS = Crisis in Family Systems Scale; AUC = area under the curve;

several weeks to months and is largely reflective of $\mathrm{MeHg}$ [37]. Previous studies have shown toenail levels of $\mathrm{Hg}$ to be stable over time [38,39], to be highly correlated with $\mathrm{Hg}$ levels in blood and hair $[39,40]$ and to be highly correlated with fish consumption $[22,37,40]$.

We performed mercury assays using the Direct Mercury Analyzer 80 (Milestone Inc., Monroe, CT). This automatic mercury analyzer requires no sample digestion or pretreatment. The cleaned sample [41] of toenail was weighed into a nickel boat, thermally decomposed, amalgamated, and the released mercury measured by atomic absorption spectroscopy at $253.7 \mathrm{~nm}$ as a function of mercury concentration. Samples were analyzed by using an aqueous calibration curve. Quality control steps included daily calibration with verification of a high and low concentration standard for each working range, a procedural blank, and Certified Reference material GBW 07601 (human hair; Institute of Geophysical and Geochemical Exploration, Langfang, Hebei Province, People's Republic of China). The detection limit (DL) for the mercury analysis was $0.5 \times 10^{-3} \mu \mathrm{g}$. The DL for the sample varied according to sample weight. Sample weight varied from $0.007 \mathrm{~g}$ to $0.081 \mathrm{~g}$ and the DL varied from $0.006 \mu \mathrm{g} / \mathrm{g}$ to $0.077 \mu \mathrm{g} / \mathrm{g}$ (mean $=0.018 \mu \mathrm{g} / \mathrm{g}$ ). Mercury recovery from the standard using this procedure was $86 \%-120 \%$, with intraday precision varying from $1.2 \%$ RSD to $9 \%$ RSD. Interday precision was $6.5 \%$. Hg values in the present sample ranged from $0.0-1.9 \mu \mathrm{g} / \mathrm{g}$.

\section{Psychosocial stress}

Participants completed the Crisis in Family Systems [42] questionnaire which has been translated and validated in
Spanish [43] to indicate stressful life events they had experienced over the past six months. The CRISYS questionnaire asks participants to indicate whether or not they experienced potentially stressful life events in 11 domains (financial; legal; career; relationships; safety in the home; safety in the community; medical issues pertaining to respondent; medical issues pertaining to close others; home issues; difficulties with authority; prejudice). In addition, participants indicated the valence of all experienced events, i.e. whether or not they perceived a certain event negatively or not. Participants were coded as having experienced any or no negative life events in each one of the 11 domains as done previously [44]. A sum score was created by counting the number of domains for which participants endorsed any negative events, resulting in possible scores from 0 (no negative events endorsed) to 11 (at least one negative event endorsed in each domain). In this sample, participants reported negative events in an average of $2.27 \pm 1.6$ domains (range $0-8$ ). The CRISYS has previously been shown to have good test-retest reliability and to be a valid measure of life events [43]. It has furthermore been linked to health outcomes in a dose-response manner, such that exposure to negative life events in multiple domains is associated with worsening health outcomes [44], and has previously been linked to disrupted cortisol rhythms [45].

\section{Diurnal cortisol}

All women collected saliva samples at home for the assessment of salivary cortisol. Saliva samples were collected when women were on average $19.52 \pm 3.00$ weeks pregnant. To capture the diurnal variation in cortisol production, participants were instructed to collect five saliva samples into tubes using the passive drool technique [46] on each of two consecutive days. Prior to sample collection, research staff instructed women to provide samples when they awoke ("when you open your eyes"), 45 minutes after waking, 4 hours after waking, 10 hours after waking, and at bedtime ("right before getting into bed"). Participants followed these instructions well, collecting their five saliva samples an average of $.13 \pm .28, .99 \pm .40,4.50 \pm .74,10.70 \pm 1.20$, and $15.21 \pm$ 1.29 hours following waking. After collecting each sample, women recorded the collection time on the tube and in a diary. Participants were instructed not to eat, brush their teeth, or drink liquids for at least 15 minutes before providing a sample and not drink caffeinated beverages before collecting the first two samples. Women were asked to refrigerate samples until pickup on the third day after which they were stored at $-70^{\circ} \mathrm{C}$ until shipment on dry ice to the laboratory of Dr. Clemens Kirschbaum in Dresden, Germany for assay. Samples were assayed in duplicates using a commercially available 
chemi-luminescence assay (IBL; Hamburg, Germany) with sensitivity of $\sim 0.16 \mathrm{ng} / \mathrm{ml}$. Intra- and interassay coefficients of variation were $<8 \%$.

Cortisol values were log-transformed to reduce skewness. Total daily cortisol output, cortisol area under the curve (AUC), was computed by calculating the area under the curve statistic using the trapezoidal rule. Specifically, for each day and each participant a line depicting cortisol at each of the five collection time points was plotted, and AUC calculated as the sum of the four trapezoids below that line. AUCs of both days were averaged. A higher AUC is suggestive of greater overall cortisol output over the course of the day. In addition, cortisol values were averaged across both collection days to increase stability and cortisol slope (cortisol values/corresponding time since wake-up) computed. Steeper slopes indicate a more rapidly declining cortisol output throughout the course of the day, flatter slopes indicate a slower decline in cortisol output over the course of the day.

\section{Potential confounders}

Participating women reported on demographic information at the time of recruitment, including age and highest level of education. Body mass index (BMI) was computed as $\mathrm{kg} / \mathrm{m}^{2}$ based on weight and height measured by a trained research assistant during a second trimester visit. All analyses also controlled for the trimester during which toe nail clippings for $\mathrm{Hg}$ assessment were collected and for weeks of gestation at the time of saliva sample collection, as cortisol levels are known to rise over the course of pregnancy [47].

\section{Statistical analyses}

Eleven $\mathrm{Hg}$ and three CRISYS scores were more than three standard deviations from the mean. Winsorization to reduce these scores to the next highest score did not change our results, however, and consequently the original values were retained for the analyses reported below. We first tested the main effects of $\mathrm{Hg}$ exposure and exposure to negative life events (controlling for the other) on maternal diurnal cortisol profiles (area under the curve, diurnal slope, and cortisol levels at individual collection time points averaged across both days and controlling for time since waking) using multiple linear regression analyses. Next, we used multiple linear regression analyses to test whether $\mathrm{Hg}$ exposure interacted with exposure to life stressors to predict maternal diurnal cortisol profiles. Following the guidelines for interaction effects provided by Aiken and West [48] $\mathrm{Hg}$ and life stress variables were centered and subsequently multiplied to create the interaction term. We first entered $\mathrm{Hg}$ exposure, negative life events, and covariates into the model, then added the interaction term between
$\mathrm{Hg}$ exposure and negative life events to examine the effect modification. Regression analyses were conducted using SPSS Statistics 20 for Windows (IBM). In addition, to visualize and enhance the interpretability of our results, treating the five $\log$ (cortisol) measures as observations from an overall nonlinear curve for each individual, we used functional mixed models with penalized splines (FMPS, [49]) using R [50] to estimate main effects of and interactions between the two exposures of interest on the mean cortisol curve for a given individual. Participants were placed into one of 4 groups indicating whether they were below the median on both $\mathrm{Hg}$ exposure and life stress (LL), below the median for $\mathrm{Hg}$ exposure and above the median for life stress (LH), above the median for $\mathrm{Hg}$ exposure and below the median for life stress (HL), or above the median for both $\mathrm{Hg}$ exposure and life stress $(\mathrm{HH})$. Time since waking was used as the time metric for each individual. Predicted diurnal cortisol profile (log(cortisol) over time (hours)) using FMPS for each group was plotted in Figure 1.

\section{Results}

Main effects of $\mathrm{Hg}$ exposure and psychosocial stress

First, we investigated the main effects of $\mathrm{Hg}$ exposure and life stress on maternal diurnal cortisol profiles. When used to predict maternal diurnal cortisol outcomes independently, $\mathrm{Hg}$ exposure was unrelated to cortisol AUC $(p>.30)$ and diurnal cortisol slope $(p>30)$. We also assessed the influence of $\mathrm{Hg}$ exposure on cortisol values at the individual times of data collection, averaged across the two days, controlling for time since waking. Maternal $\mathrm{Hg}$ exposure was unrelated to cortisol at the first four collection times over the course of the day (all $p s>.50$ ) but marginally associated with lower cortisol at time 5 , i.e. before bedtime $(\mathrm{B}=-.080, \mathrm{SE}=.044, p=.070)$.

Exposure to stressful life events, when $\mathrm{Hg}$ is also entered into the model, was also unrelated to cortisol AUC $(p>.60)$ and diurnal slope $(p>.60)$. Report of more stressful life events was associated with marginally higher cortisol at the first collection time of the day, i.e. immediately following waking $(\mathrm{B}=.007, \mathrm{SE}=.004$, $\mathrm{p}=.075)$. Exposure to stressful life events was unrelated to cortisol levels at all other collection times throughout the day (all $p \mathrm{~s}>.10$ ).

See Table 2 for details regarding our main effects analyses.

\section{Interaction effects of $\mathrm{Hg}$ and psychosocial stress}

Second, we investigated whether exposure to negative life events and $\mathrm{Hg}$ exposure interacted to influence maternal diurnal cortisol. There was a significant $\mathrm{Hg} \mathrm{x}$ negative life events interaction effect on maternal cortisol slope (interaction $\mathrm{B}=.006, \mathrm{SE}=.003, p=.033$ ) but not maternal cortisol AUC $(p>30)$. As can be seen in Figure 1 (panel a), there is no effect of $\mathrm{Hg}$ exposure on 


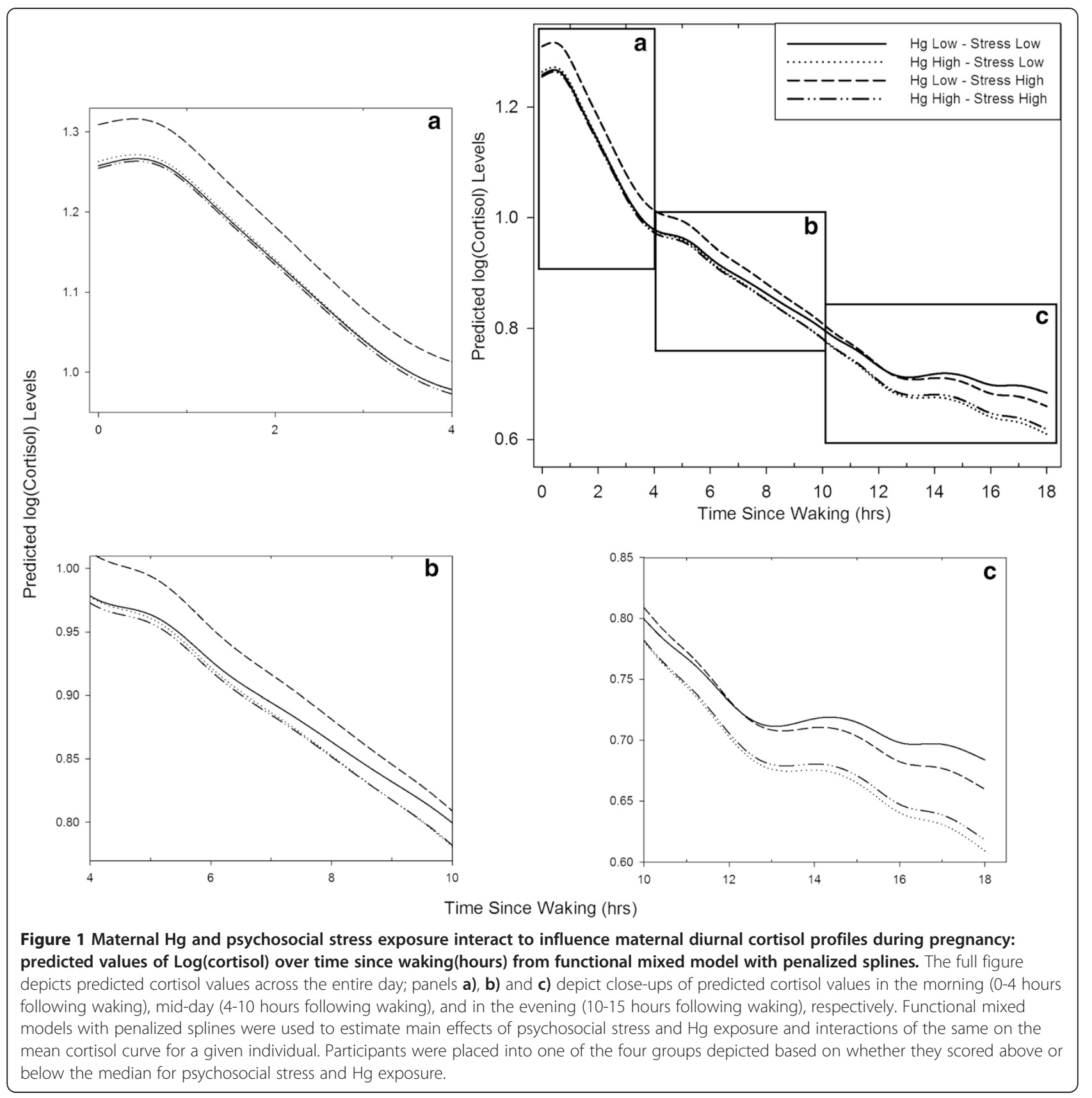

cortisol in the low stress group; however, in the high stress group, there is a negative effect of $\mathrm{Hg}$ exposure such that the stress response (increased cortisol) is blunted among women exposed to $\mathrm{Hg}$ levels above the median. Conversely, women in the high stress group who are below the median for $\mathrm{Hg}$ exposure show evidence of greater cortisol levels in the morning. Panels b) and c) of Figure 1 show that this negative effect of $\mathrm{Hg}$ exposure among the higher stress group weakens later in the day. When considering the $\mathrm{Hg} \mathrm{x}$ life stress interaction at individual cortisol collection time points throughout the day, there also were significant negative effects at time 1 , i.e. immediately following waking (interaction $\mathrm{B}=-.071, \mathrm{SE}=.028, \mathrm{p}=.013$ ), and time 2, i.e. approximately one hour following waking (interaction $\mathrm{B}=-.078, \mathrm{SE}=.032, \mathrm{p}=.014$ ); see Table 2 . Cortisol levels at later times of the day were not significantly associated with the $\mathrm{Hg} \times$ life stress interaction term (all $p s>.20$ ). Figure 2, a graphical depiction of the results from FMPS, shows that the interaction effect is significant during the first two hours following waking, further suggesting that it is the early morning differences that 
Table 2 Regression analyses of main and interaction effects between $\mathrm{Hg}$ and psychosocial stress exposure

\begin{tabular}{|c|c|c|c|c|}
\hline Outcome & Model & $\begin{array}{l}\text { Psychosocial stress (CRISYS) } \\
\text { [B (SE, p)] }\end{array}$ & $\begin{array}{l}\mathrm{Hg} \\
{[B(S E, p)]}\end{array}$ & $\begin{array}{l}\text { CRISYS x Hg } \\
{[B(S E, p)]}\end{array}$ \\
\hline \multirow[t]{2}{*}{$\overline{A \cup C}$} & Main effects & $.026(.057, .649)$ & $-.536(.596, .369)$ & - \\
\hline & Interaction effects & $.027(.057, .640)$ & $-.640(.606, .292)$ & $-.400(.420, .341)$ \\
\hline \multirow[t]{2}{*}{ Slope } & Main effects & $.000(.000, .622)$ & $-.004(.004, .304)$ & - \\
\hline & Interaction effects & $.000(.000, .604)$ & $-.002(.004, .529)$ & $.006(.003, .033)$ \\
\hline \multirow[t]{2}{*}{ Time 1} & Main effects & $.007(.004, .075)$ & $-.010(.041, .798)$ & - \\
\hline & Interaction effects & $.007(.004, .070)$ & $-.029(.041, .481)$ & $-.071(.028, .013)$ \\
\hline \multirow[t]{2}{*}{ Time 2} & Main effects & $.006(.004, .198)$ & $.026(.046, .561)$ & - \\
\hline & Interaction effects & $.006(.004, .188)$ & $.006(.046, .898)$ & $-.078(.032, .014)$ \\
\hline \multirow[t]{2}{*}{ Time 3} & Main effects & $.003(.004, .451)$ & $-.024(.041, .558)$ & - \\
\hline & Interaction effects & $.003(.004, .446)$ & $-.033(.042, .432)$ & $-.033(.029, .251)$ \\
\hline \multirow[t]{2}{*}{ Time 4} & Main effects & $.002(.004, .632)$ & $-.028(.045, .535)$ & - \\
\hline & Interaction effects & $.002(.004, .634)$ & $-.026(.045, .569)$ & $.007(.031, .822)$ \\
\hline \multirow[t]{2}{*}{ Time 5} & Main effects & $.002(.004, .620)$ & $-.080(.044, .070)$ & - \\
\hline & Interaction effects & $.002(.004, .624)$ & $-.077(.045, .086)$ & $.011(.031, .721)$ \\
\hline
\end{tabular}

$\mathrm{AUC}=$ area under the curve; $\mathrm{Hg}=$ mercury; CRISYS = Crisis in Family Systems Scale;

Note: All analyses controlled for maternal age, education, body mass index, weeks gestation at the time of saliva collection, and the pregnancy trimester during which toe nails were collected for $\mathrm{Hg}$ assessment. Analyses investigating the influence of $\mathrm{Hg}$ and psychosocial stress on cortisol levels at individual time points additionally controlled for time since waking. Significant findings are highlighted in bold.

affect the diurnal slope (flatter among women exposed to greater psychosocial stress and $\mathrm{Hg}$ levels compared to women exposed to greater psychosocial stress but lower levels of $\mathrm{Hg}$ ).

\section{Discussion}

This study is among the first to examine the synergistic influence of toxic exposures in the physical environment, i.e. $\mathrm{Hg}$, and in the social environment, i.e. negative life events, on human HPA axis activity. In addition, in the present study we focused on examining these associations among pregnant women, as cortisol production during pregnancy is increasingly being linked to important developmental outcomes among offspring [2,4]. Environmental toxicants with the potential to alter maternal cortisol output during pregnancy hence may have important consequences for the healthy development of offspring. Toxicants that commonly occur jointly with stressful life events need to be better understood. Our findings highlight the importance of considering physical and social environmental influences jointly, rather than independently, as their synergistic effects may have a stronger influence on physiological outcomes of interest, including HPA activity in the present study. In addition, with regards to health disparities, the joint occurrence of stressful life events and toxic chemicals may be most common in economically disadvantaged populations.

As part of the present study we examined both main effects and interaction effects of $\mathrm{Hg}$ and psychosocial stress exposure. We found that, in a main effects model, maternal prenatal $\mathrm{Hg}$ exposure was marginally negatively associated with cortisol levels before bedtime (though not at other times). We found no main effects of negative life events, although exposure to more negative life events was associated with marginally higher cortisol levels in the morning. These findings are in line with previous research that has linked the experience of greater stress to a greater increase in cortisol following waking [51] and recent research linking even low-level Hg exposure among children to a blunted diurnal cortisol response to stress [52]. When considering synergistic influences of the social and physical environment we found that among women exposed to high levels of psychosocial stress (more negative life events), simultaneous exposure to greater $\mathrm{Hg}$ levels resulted in a blunted cortisol response early in the day compared to women experiencing greater psychosocial stress but lower levels of $\mathrm{Hg}$ exposure.

These findings are also in line with limited evidence from animal studies suggesting that $\mathrm{Hg}$ exposure can disrupt HPA functioning, leading to, for example, reduced cortisol levels in catfish [53]. A small number of studies investigating the effect of $\mathrm{Hg}$ exposure among human adults have found no impact of $\mathrm{Hg}$ exposure on HPA axis functioning $[54,55]$; however, these studies are limited by their small sample sizes and the indices of HPA axis functioning used, specifically, one-time assessments of serum cortisol levels which do not allow for the evaluation of daily cortisol rhythms. Existing research in fish further supports the idea that psychosocial stress and $\mathrm{Hg}$ exposure interact. Miller et al. [19] found 


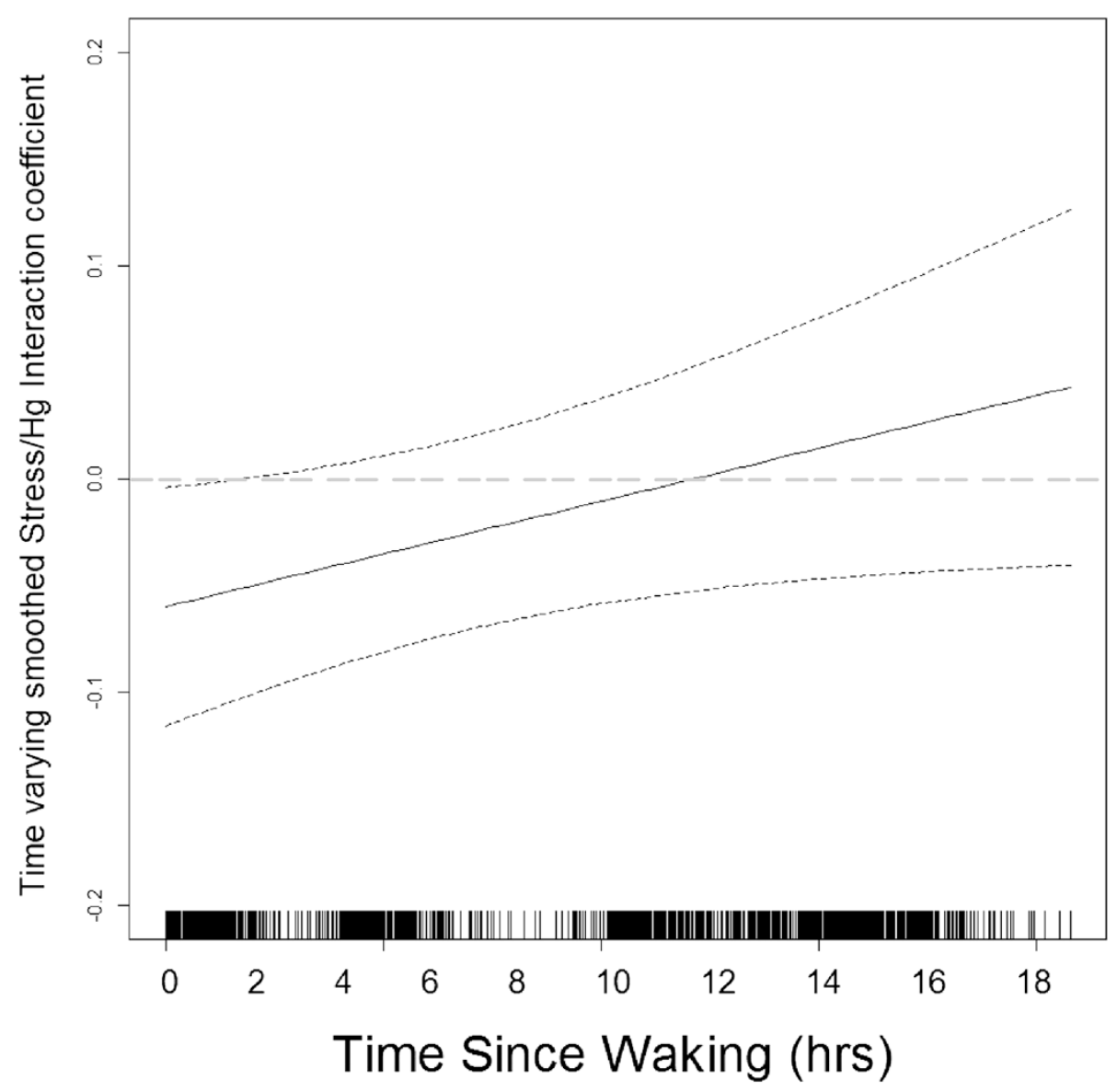

Figure 2 Maternal $\mathrm{Hg}$ and psychosocial stress exposure interact to influence maternal diurnal Cortisol profiles during pregnancy: time-varying interaction beta. The graph of the time-varying interaction beta shows a significant, negative interaction between $\mathrm{Hg}$ and psychosocial stress exposure during the first two hours of the day. Early morning differences in cortisol (during the first two hours following waking) result in flatter slopes among women exposed to greater psychosocial stress and Hg levels compared to women exposed to greater psychosocial stress but lower levels of $\mathrm{Hg}$.

that when treating lake trout thymocytes with either $\mathrm{Hg}$ alone or $\mathrm{Hg}$ and cortisol together, the presence of cortisol increased the toxicity of $\mathrm{Hg}$. In addition, one study investigating yellow perch and northern pike provides further evidence of the potential synergistic effects of $\mathrm{Hg}$ exposure and stress. Hontela et al. [56] found that fish from polluted sites (including, but not restricted to, $\mathrm{Hg}$ pollution) failed to show the typical cortisol response to capture that was observed in control fish from unpolluted areas, again suggesting blunted cortisol responses in response to $\mathrm{Hg}$ exposure.

Strengths of the present study include the large sample size relative to nearly all other reports of chemical exposure on cortisol as well as the collection of saliva samples across five time points on each of two days, allowing for more stable assessments of daily cortisol rhythms. In addition, we were able to assess $\mathrm{Hg}$ levels in toe nail clippings collected during pregnancy, providing us with a measure of longer-term $\mathrm{Hg}$ exposure across several months. Our population is not coastal and is not high in fish consumption, thus it is interesting that we see an association between $\mathrm{Hg}$ exposure and diurnal cortisol patterns at these relatively low levels of $\mathrm{Hg}$ exposure, suggesting that our findings may be conservative. Future studies should investigate these associations among populations exposed to higher levels of $\mathrm{Hg}$.

Although we were able to evaluate the association of simultaneous exposure to $\mathrm{Hg}$ and negative life events and diurnal cortisol slope, an indicator of the diurnal rhythm of cortisol production, it is possible that $\mathrm{Hg}$ exposure and exposure to negative life events might be differentially associated with cortisol patterns in response to acute stressors. Future research should investigate whether acute stress responses are altered as a function of these exposures. Similarly, the present study employed a summary score representing different types of psychosocial stressors experienced over a 6-month period. It is unclear whether certain types of stressors, e. 
g. stressors directly impacting home rather than work life, are more likely to interact with $\mathrm{Hg}$ and result in potentially adverse physiological outcomes. Due to sample size restrictions we were not able to investigate interaction effects with individual negative life stress domains as part of the present study. However, as previously mentioned, existing research suggests a dose-response relation between exposure to negative life events across multiple domains and adverse health outcomes [44] and has linked the CRISYS to altered cortisol rhythms [45]. As such exposure may place increased burden on individuals and result in greater distress, considering $\mathrm{cu}-$ mulative stress exposure may be valuable. It is also important to note that this study was not experimental and that, consequently, alternative explanations for our findings cannot be ruled out. For example, the exact effects of $\mathrm{Hg}$ exposure on human HPA-axis functioning is not clearly understood, nor are the possible effects of diurnal cortisol on $\mathrm{Hg}$ toxicokinetics. Hence, our findings must be viewed with caution until the underlying mechanisms linking $\mathrm{Hg}$ and cortisol in humans are better understood. In addition, we were only able to measure total $\mathrm{Hg}$ in the present study. Although total $\mathrm{Hg}$ has been shown to be largely reflective of $\mathrm{MeHg}$ [37], it cannot be ruled out that our findings are the result of an interaction between exposure to psychosocial stressors and levels of inorganic $\mathrm{Hg}$ and this needs to be investigated further. Due to the use of an entirely Mexican birth cohort, the generalizability of the present findings are also limited. However, both the levels of $\mathrm{Hg}$ found in toe nails and the scores on the CRISYS questionnaire are comparable to those found in American samples [57], suggesting that similar associations may exist among non-Mexican populations as well. Finally, future studies should also examine the combined impact of maternal $\mathrm{Hg}$ exposure and life stress during pregnancy on relevant developmental outcomes among their offspring, such as birth weight and indicators of cognitive development.

\section{Conclusion}

This study suggests that physical and social environmental influences interact to alter aspects of maternal diurnal cortisol profiles, an indicator of HPA axis activity, among a sample of pregnant women in Mexico City. Specifically, among women exposed to more negative life events, exposure to higher levels of $\mathrm{Hg}$ resulted in a blunted salivary cortisol response early in the day. With respect to research focusing on environmental effects on pregnancy outcomes or developmental effects among offspring, these findings suggest that conducting research focusing solely on either physical or social pollutants may miss important synergistic associations that have the potential to negatively impact the healthy development of offspring.

\section{Abbreviations}

BMI: Body mass index; Hg: Mercury; HPA: Hypothalamic-pituitary-adrenal; MeHg: Methylmercury.

\section{Competing interests}

The authors declare that they have no competing interests.

\section{Authors' contributions}

HMCS; data analysis, manuscript drafting. H-HS; data analysis. CA; sample processing and analysis. BAC; data analysis. LS; study design and coordination. MMT-R; study design and coordination. MTO; study coordination. RJW; stress assessment, manuscript drafting. ROW; study design and coordination, manuscript drafting. All authors have read and approved the final manuscript.

\section{Acknowledgements}

This study was funded by R01 ES013744; R01ES014930; R01ES021357; P30 ES023515. We thank the ABC Hospital in Mexico City for providing research facilities.

\section{Author details}

${ }^{1}$ Department of Pediatrics, Icahn School of Medicine at Mount Sinai, One Gustave L. Levy Place, Box 1198, New York, NY 10029, USA. ²Department of Preventive Medicine, Icahn School of Medicine at Mount Sinai, New York, NY, USA. ${ }^{3}$ Department of Biostatistics, Harvard School of Public Health, Boston, MA, USA. ${ }^{4}$ Department of Environmental Health, Harvard School of Public Health, Boston, MA, USA. ${ }^{5}$ Division of Research on Public Health, National Institute of Perinatology, Mexico City, Mexico. ${ }^{6}$ Center for Evaluation Research and Surveys, National Institute of Public Health, Cuernavaca, Morelos, Mexico. ${ }^{7}$ Center for Research in Nutrition and Health, National Institute of Public Health, Cuernavaca, Morelos, Mexico.

Received: 23 October 2014 Accepted: 10 March 2015

Published online: 27 March 2015

\section{References}

1. Van Cauter E. Endocrine rhythms. In: Becker KL, editor. Principles and Practice of Endocrinology and Metabolism. Philadelphia: J. B. Lippincott Company; 1995. p. 41-50.

2. Wadhwa PD. Psychoneuroendocrine processes in human pregnancy influence fetal development and health. Psychoneuroendocrinology. 2005;30:724-43.

3. Weinstock $M$. The potential influence of maternal stress hormones on development and mental health of the offspring. Brain Behav Immun. 2005;19:296-308.

4. O'Donnell K, O'Connor TG, Glover V. Prenatal stress and neurodevelopment of the child: focus on the HPA axis and role of the placenta. Dev Neurosci. 2009;31:285-92.

5. Cory-Slechta DA, Virgolini MB, Rossi-George A, Thiruchelvam M, Lisek R, Weston D. Lifetime consequences of combined maternal lead and stress. Basic Clin Pharmacol Toxicol. 2008;102:218-27.

6. Gump BB, Stewart P, Reihman J. Low-level prenatal and postnatal blood lead exposure and adrenocortical responses to acute stress in children. Environ Health Perspect. 2008;116:249.

7. Levine S. Influence of psychological variables on the activity of the hypothalamic-pituitary-adrenal axis. Eur J Pharmacol. 2000;405:149-60.

8. Miller GE, Chen E, Zhou ES. If it goes up, must it come down? Chronic stress and the hypothalamic-pituitary-adrenocortical axis in humans. Psychol Bull. 2007;133:25

9. Dickerson SS, Kemeny ME. Acute stressors and cortisol responses: a theoretical integration and synthesis of laboratory research. Psychol Bull. 2004;130:355.

10. Diego MA, Jones NA, Field T, Hernandez-Reif M, Schanberg S, Kuhn C, et al. Maternal psychological distress, prenatal cortisol, and fetal weight. Psychosom Med. 2006;68:747-53.

11. Obel C, Hedegaard M, Henriksen TB, Secher NJ, Olsen J, Levine S. Stress and salivary cortisol during pregnancy. Psychoneuroendocrinology. 2005;30:647-56.

12. Pluess $M$, Bolten $M$, Pirke K-M, et al. Maternal trait anxiety, emotional distress, and salivary cortisol in pregnancy. Biol Psychol. 2010;83:169-75.

13. Bolten Ml, Wurmser H, Buske-Kirschbaum A, Papusek M, Pirke KM, Hellhammer D. Cortisol levels in pregnancy as a psychobiological predictor for birth weight. Arch Womens Ment Health. 2011;14:33-41. 
14. LeWinn KZ, Stroud LR, Molnar BE, Ware JH, Koenen KC, Bulka SL. Elevated maternal cortisol levels during pregnancy are associated with reduced childhood IQ. Int J Epidemiol. 2009;38:1700-10.

15. Wright RJ, Fisher K, Chiu YH, Wright RO, Fein R, Cohen S, et al. Disrupted prenatal maternal cortisol, maternal obesity, and childhood wheeze. Insights into prenatal programming. Am J Respir Crit Care Med. 2013;187:1186-93.

16. Tan SW, Meiller JC, Mahaffey KR. The endocrine effects of mercury in humans and wildlife. Crit Rev Toxicol. 2009;39:228-69.

17. Brkljacic J, Milutinovic DV, Dundjerski J, Matic G. Mercury stimulates rat liver glucocorticoid receptor association with $\mathrm{Hsp90}$ and Hsp70. J Biochem Mol Toxicol. 2004;18:257-60.

18. Brkljacic J, Vojnovic Milutinovic D, Dundjerski J, Matic G. Mercury inhibits rat liver and kidney glucocorticoid receptor hormone binding activity. Cell Biol Toxicol. 2004;20:171-82.

19. Miller GG, Sweet LI, Adams JV, Omann GM, Passino-Reader DR, Meier PG. i> In vitro toxicity and interactions of environmental contaminants (Arochlor 1254 and mercury) and immunomodulatory agents (lipopolysaccharide and cortisol) on thymocytes from lake trout ( $<$ i $>$ Salvelinus namaycush). Fish Shellfish Immunol. 2002;13:11-26.

20. Wada H, Yates DE, Evers DC, Taylor RJ, Hopkins WA. Tissue mercury concentrations and adrenocortical responses of female big brown bats (Eptesicus fuscus) near a contaminated river. Ecotoxicology. 2010;19:1277-84.

21. Clarkson TW, Vyas JB, Ballatori N. Mechanisms of mercury disposition in the body. Am J Ind Med. 2007;50:757-64.

22. Xun P, Liu K, Morris JS, Jordan JM, He K. Distributions and determinants of mercury concentrations in toenails among American young adults: the CARDIA Trace Element Study. Environmental Science and Pollution Research. 2013;20(3):1423-30.

23. Fernandes Azevedo B, Barros Furieri L, Peçanha FM, Wiggers GA, Frizera Vassallo P, Ronacher Simoes M, et al. Toxic effects of mercury on the cardiovascular and central nervous systems. J BioMed Biotechnol. 2012;2012:949048. doi: 10.1155/2012/949048

24. Holmes $P$, James KAF, Levy LS. Is low-level environmental mercury exposure of concern to human health? Sci Total Environ. 2009;408:171-82.

25. Davidson PW, Kost J, Myers GJ, Cox C, Clarkson TW, Shamlaye CF. Methylmercury and neurodevelopment: reanalysis of the Seychelles Child Development Study outcomes at 66 months of age. JAMA. 2001;285:1291-3.

26. Myers GJ, Davidson PW, Cox C, Shamlaye CF, Palumbo D, Cernichiari E, et al. Prenatal methylmercury exposure from ocean fish consumption in the Seychelles child development study. Lancet. 2003;361:1686-92.

27. Grandjean P, Weihe P, White RF, Debes F, Araki S, Yokoyama K, et al. Cognitive deficit in 7-year-old children with prenatal exposure to methylmercury. Neurotoxicol Teratol. 1997;19:417-28.

28. Gee GC, Takeuchi DT. Traffic stress, vehicular burden and well-being: a multilevel analysis. Soc Sci Med. 2004;59:405-14.

29. Morello-Frosch R, Shenassa ED. The environmental "riskscape" and social inequality: implications for explaining maternal and child health disparities. Environ Health Perspect. 2006;114:1150-3.

30. Weiss B, Bellinger DC. Social ecology of children's vulnerability to environmental pollutants. Environ Health Perspect. 2006;114:1479-85.

31. Surkan PJ, Schnaas L, Wright RJ, Tellez-Rojo MM, Lamadrid-Figueroa $H_{4}$ $\mathrm{Hu} \mathrm{H}$, et al. Maternal self-esteem, exposure to lead, and child neurodevelopment. Neurotoxicology. 2008;29:278-85.

32. Schreier HM, Chen E. Socioeconomic status and the health of youth: a multilevel, multidomain approach to conceptualizing pathways. Psychol Bull. 2013;139:606-54.

33. Wright RJ. Moving towards making social toxins mainstream in children's environmental health. Curr Opin Pediatr. 2009;21:222-9.

34. Rossi-George A, Virgolini MB, Weston D, Cory-Slechta DA. Alterations in glucocorticoid negative feedback following maternal $\mathrm{Pb}$, prenatal stress and the combination: a potential biological unifying mechanism for their corresponding disease profiles. Toxicol Appl Pharmacol. 2009;234:117-27.

35. Virgolini MB, Rossi-George A, Weston D, Cory-Slechta DA, et al. Influence of low level maternal $\mathrm{Pb}$ exposure and prenatal stress on offspring stress challenge responsivity. Neurotoxicology. 2008;29:928-39.

36. Gump BB, Reihman J, Stewart P, Lonky E, Granger DA, Matthews KA. Blood lead (Pb) levels: further evidence for an environmental mechanism explaining the association between socioeconomic status and psychophysiological dysregulation in children. Health Psychol. 2009;28:614-20.
37. Rees JR, Sturup S, Chen C, Folt C, Karagas MR. Toenail mercury and dietary fish consumption. Journal of Exposure Science and Environmental Epidemiology. 2006;17:25-30

38. Garland M, Morris JS, Rosner BA, Stampfer MJ, Spate VL, Baskett CJ, et al. Toenail trace element levels as biomarkers: reproducibility over a 6-year period. Cancer Epidemiol Biomarkers Prev. 1993;2:493-7.

39. Hinners T, Tsuchiya A, Stern AH, Burbacher TM, Faustman EM, Marien K. Chronologically matched toenail-Hg to hair-Hg ratio: temporal analysis within the Japanese community (US). Environ Health. 2012;11:81.

40. Björkman L, Lundekvam BF, Lægreid T, Bertelsen Bl, Morild I, Lilleng P, et al. Mercury in human brain, blood, muscle and toenails in relation to exposure: an autopsy study. Environ Health. 2007;6:13.

41. Mordukhovich I, Wright $\mathrm{RO}, \mathrm{Hu} H$, Amarasiriwardena C, Baccarelli A, Litonjua A, et al. Associations of toenail arsenic, cadmium, mercury, manganese, and lead with blood pressure in the normative aging study. Environ Health Perspect. 2012;120:98-104.

42. Shalowitz MU, Berry CA, Rasinski KA, Dannhausen-Brun CA. A new measure of contemporary life stress: development, validation, and reliability of the CRISYS. Health Serv Res. 1998;33:1381-402.

43. Berry CA, Quinn KA, Portillo N, Shalowitz MU. Reliability and validity of the Spanish Version of the Crisis in Family Systems-Revised. Psychol Rep. 2006;98:123-32.

44. Chiu YHM, Coull BA, Cohen S, Wooley A, Wright RJ. Prenatal and postnatal maternal stress and wheeze in urban children: effect of maternal sensitization. Am J Respir Crit Care Med. 2012;186:147-54.

45. Suglia SF, Staudenmayer J, Cohen S, Enlow MB, Rich-Edwards JW, Wright RJ. Cumulative Stress and Cortisol Disruption among Black and Hispanic Pregnant Women in an Urban Cohort. Psychol Trauma. 2010;2:326-34.

46. Strazdins L, Meyerkort S, Brent V, D'Souza V, Broom DH, Kyd JM. Impact of saliva collection methods on slgA and cortisol assays and acceptability to participants. J Immunol Methods. 2005;307:167-71.

47. Kirschbaum C, Tietze A, Skoluda N, Dettenborn L. Hair as a retrospective calendar of cortisol production-increased cortisol incorporation into hair in the third trimester of pregnancy. Psychoneuroendocrinology. 2009;34:32-7.

48. Aiken LS, West SG. Multiple regression: Testing and interpreting interactions. Thousand Oaks, CA: Sage; 1996.

49. Sanchez BN, Wu M, Raghunathan TE, Diez-Roux AV. Modeling the salivary cortisol profile in population research: the multi-ethnic study of atherosclerosis. Am J Epidemiol. 2012;176:918-28.

50. Team RC. R: A language and environment for statistical computing, reference index version 3.0.0. 2013

51. Fries $E$, Dettenborn L, Kirschbaum $C$. The cortisol awakening response (CAR): facts and future directions. Int J Psychophysiol. 2009;72:67-73.

52. Gump BB, MacKenzie JA, Dumas AK, Palmer CD, Parsons PJ, Sego ZM, et al. Fish consumption, low-level mercury, lipids, and inflammatory markers in children. Environ Res. 2012;112:204-11.

53. Kirubagaran R, Joy KP. Changes in adrenocortical-pituitary activity in the catfish, $<\mathrm{i}>$ Clarias batrachus(L.), after mercury treatment. Ecotoxicol Environ Saf. 1991;22:36-44.

54. Erfurth EM, Schütz A, Nilsson A, Barregard L, Skerfving S. Normal pituitary hormone response to thyrotrophin and gonadotrophin releasing hormones in subjects exposed to elemental mercury vapour. $\mathrm{Br} \mathrm{J}$ Ind Med. 1990:47:639-44.

55. Langworth S, Röjdmark S, Akesson A. Normal pituitary hormone response to thyrotrophin releasing hormone in dental personnel exposed to mercury. Swed Dent J. 1990;14:101.

56. Hontela A, Rasmussen JB, Audet C, Chevalier G. Impaired cortisol stress response in fish from environments polluted by PAHs, PCBs, and mercury. Arch Environ Contam Toxicol. 1992;22:278-83.

57. Peters JL, Cohen S, Staudenmayer J, Hosen J, Platts-Mills TAE, Wright RJ. Prenatal negative life events increases cord blood lgE: interactions with dust mite allergen and maternal atopy. Allergy. 2012;67:545-51. 